\title{
Feline and canine coronaviruses are released from the basolateral side of polarized epithelial LLC-PK1 cells expressing the recombinant feline aminopeptidase-N cDNA
}

\author{
Brief Report
}

\author{
J. W. A. Rossen ${ }^{1,2}$, J. Kouame ${ }^{1}$, A. J. W. Goedheer ${ }^{1}$, H. Vennema ${ }^{1}$, and P. J. M. Rottier ${ }^{1}$ \\ ${ }^{1}$ Institute of Virology, Department of Infectious Diseases and Immunology, Faculty \\ of Veterinary Medicine, Utrecht University, Utrecht, The Netherlands \\ ${ }^{2}$ Pediatric Gastroenterology and Nutrition, Erasmus University Medical Center, \\ Rotterdam, The Netherlands
}

Accepted November 24, 2000

Summary. In this study feline (FECV and FIPV) and canine (CCoV) coronavirus entry into and release from polarized porcine epithelial LLC-PK1 cells, stably expressing the recombinant feline aminopeptidase- $\mathrm{N}$ cDNA, were investigated. Virus entry appeared to occur preferentially through the apical membrane, similar to the entry of the related porcine coronavirus transmissible gastroenteritis virus (TGEV) into these cells. However, whereas TGEV is released apically, feline and canine coronaviruses were found to be released from the basolateral side of the epithelial cells. These observations indicate that local infections as caused by $\mathrm{TGEV}, \mathrm{FECV}$ and $\mathrm{CCoV}$ do not strictly correlate with apical release, as suggested by earlier work.

$$
*
$$

Coronaviruses have been shown to infect humans and animals. The viruses have a narrow host range and the consequences of infection range from subclinical to lethal, symptoms including respiratory and enteric disease (most commonly) as well as hepatitis, peritonitis and encephalomyelitis $[5,6]$. The primary target cells of coronaviruses are the epithelial cells of the respiratory and/or gastrointestinal tract. Epithelial cells are organized in a layer and have a polarized organization, i.e., they have an apical side facing the external environment and a basolateral side facing the inner environment $[17,18]$. Previously, we have shown that coronaviruses, after being assembled intracellularly, are released from a specific side of these cells depending on the virus and the cells. Thus, transmissible gastroenteritis virus (TGEV) was released from the apical side of porcine kidney LLC-PK1 cells 
[8], whereas mouse hepatitis virus (MHV) was released basolaterally from murine kidney epithelial cells (mTAL; [9]) as well as from LLC-PK1 [10] and from human colon carcinoma cells (Caco-2; [11]) both stably expressing the recombinant MHV receptor cDNA. We suggested that the apical release of TGEV and the basolateral release of MHV might be factors contributing to the difference in pathogenesis found between TGEV and MHV in their respective natural hosts, the former causing mainly a localized enteric infection, the latter spreading through the body to other organs. However, MHV appeared to be released from the apical membrane when tested in canine kidney cells (MDCK) that stably expressed the recombinant $\mathrm{MHV}$ receptor cDNA [11], an observation incompatible with our hypothesis.

To find out whether this was just an exception, we extended our studies to include feline coronaviruses (FCoVs) and canine coronavirus $(\mathrm{CCoV})$ that, unlike $\mathrm{MHV}$, belong to the same coronavirus group as TGEV [4]. Of these viruses, CCoV and feline enteric coronavirus (FECV) cause local, enteric infections whereas feline infectious peritonitis virus (FIPV) affects many organs usually resulting in fatal peritonitis.

Because no well-characterized epithelial cell line was available to us that can be infected by these viruses, porcine kidney cells (LLC-PK1) cells were made susceptible by introducing the virus receptor. To this end, LLC-PK1 cells were transfected with pCR3-fAPN (a kind gift of Dr. Kay Holmes, Department of Microbiology, University of Colorado Health Sciences Center, Colorado, USA) containing the neomycin resistance gene and the recombinant feline aminopeptidase $\mathrm{N}$ (fAPN) cDNA. The latter encodes the protein that has been shown to function as a receptor for feline, canine, porcine and human coronaviruses. G418-resistant colonies were propagated and functionally tested for fAPN expression by assessing their susceptibility to FIPV (results not shown). One of the positive cell lines was selected for further experimentation and was named PKFA-2. This cell line remained susceptible to FIPV infection for at least 20 passages. PKFA-2 cells were grown on glass coverslips and infected with FIPV strain 1146, FECV strain 1683, CCoV strain I-71 or with the Purdue strain of TGEV. At $6 \mathrm{~h}$ p.i. cells were fixed with ice-cold methanol and prepared for indirect immunofluorescence analysis using for TGEV a porcine anti-TGEV serum (a kind gift of Dr. Luis Enjuanes, Centro Nacional de Biotecnologia, CSIC, Universidad Autónoma, Canto Blanco, Madrid, Spain) and for FCoVs and CCoV a combination (1:1) of antiserum G73 [15] and ascitic fluid A40, both obtained from experimentally FIPV-infected cats. The

Fig. 1. Susceptibility of PKFA-2 cells to CCoV, FECV, FIPV and TGEV infection. To prepare a cell line stably expressing the FIPV receptor glycoprotein, LLC-PK1 cells were transfected with pCR3-fAPN, a plasmid containing the feline APN receptor gene [14]. G418resistant cells were cloned by three rounds of limiting dilution. Cells from a selected clone (named PKFA-2) and LLC-PK1 cells were grown on coverslips and infected with the different viruses. At $6 \mathrm{~h}$ p.i. cells were fixed and processed for immunofluorescence analysis as described in the text. The different virus/cell combinations are indicated 


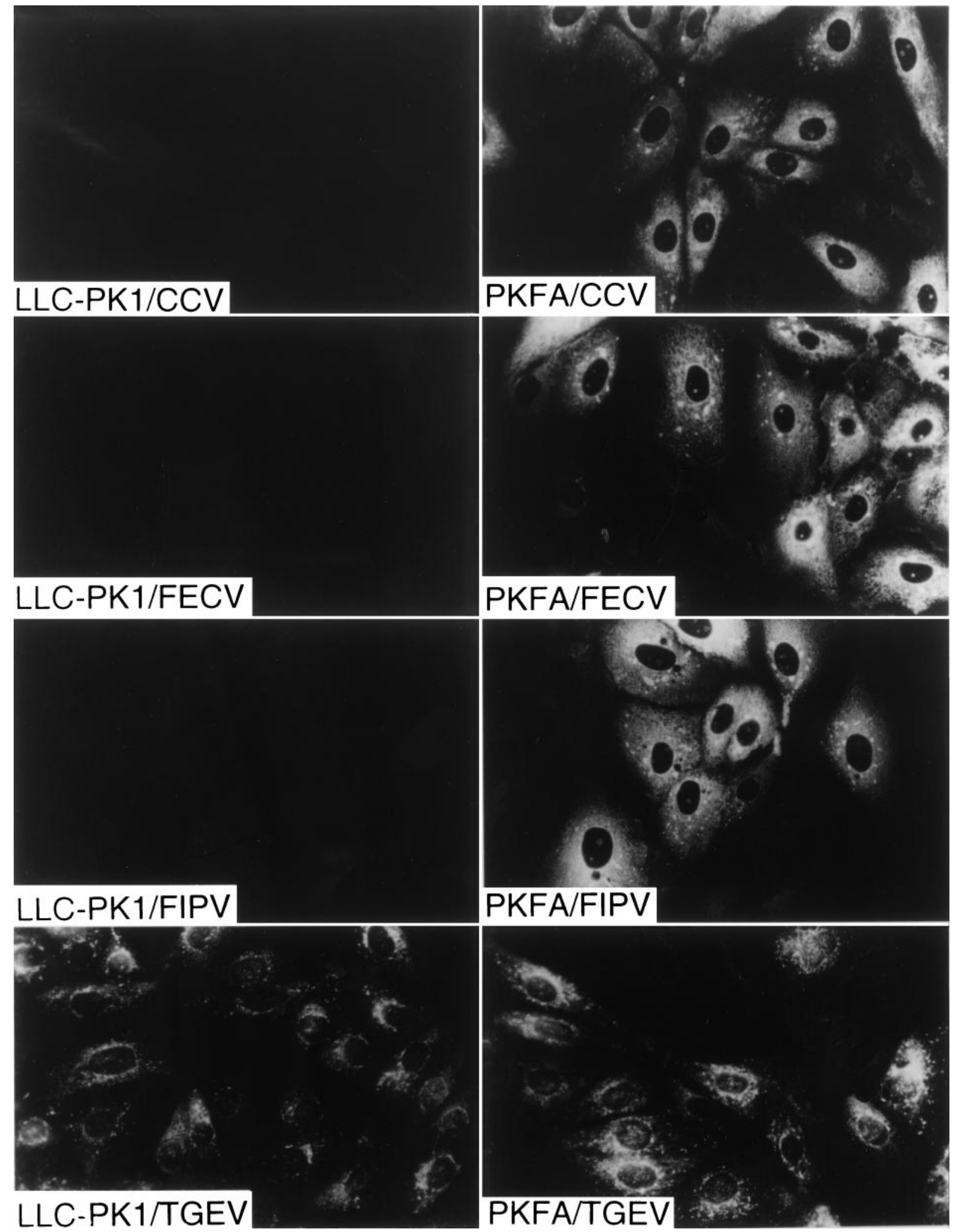


combination of A40/G73 cross-reacts with the structural proteins of FECV, CCoV and TGEV, although the spike protein of the latter was not recognized. The immunofluorescence observations of Fig. 1 show that the PKFA-2 cells supported FIPV, FECV and CCoV infection while having maintained their susceptibility to TGEV. From this experiment, and others where the m.o.i. was varied, it appeared that in each case essentially all cells could be infected by the different viruses.

To study the polarity of entry of FCoVs, CCoV and TGEV into PKFA-2 cells, monolayers of the cells were grown on permeable filters as described before $[8,9]$. This allowed inoculation both from the apical and from the basolateral side. Routinely, tightness of the monolayer was checked by adding medium to the apical compartment up to a slightly higher level than in the basolateral compartment [2]. No leakage of culture medium from the apical to the basolateral compartment occurred from $24 \mathrm{~h}$ after cells were seeded on filters and before $11 \mathrm{~h}$ post infection (p.i.). From this observation and previous experiences we decided to finish all further experiments before $9 \mathrm{~h}$ p.i. The filter-grown cells were infected from either side with the different viruses at an m.o.i. of 5 and at different times post seeding (p.s.). At $6 \mathrm{~h}$ p.i. cells were fixed with ice-cold methanol and processed for an indirect immunofluorescence assay as described previously $[8,9]$ using the antibodies mentioned above. It was found that at $1 \mathrm{~d}$ p.s. all viruses enter the epithelial cells both through the apical and the basolateral side with the same efficiency (results not shown), indicating that the filter was not a physical barrier for the viruses. However, from $2 \mathrm{~d}$ p.s. the entry started to become polarized and at $7 \mathrm{~d}$ p.s. FECV, FIPV, CCoV and TGEV entered the cells preferentially through the apical side (Fig. 2) as was found before for MHV and TGEV $[8,9]$.

The main purpose of this study was to determine the release of FIPV, FECV and CCoV from epithelial cells. Therefore, parallel cultures of PKFA-2 cells were grown on filters and infected with either virus from the apical side. Apical and basolateral culture media were analysed for the appearance of viral proteins and infectious viral particles. It is of note that, for technical reasons discussed previously [9], monolayers were used at two days of age. As we observed earlier, the kinetics with which the monolayers developed their polarity of viral entry was delayed as compared with their development of tight junctions and polarity of virus release $[8,9]$. Thus, selectivity in the release of viruses occurs already while that of entry has not yet been fully established. In the experiment shown in Fig. 3 infected cells were radiolabelled with ${ }^{35} \mathrm{~S}$-amino acids from 5-8 h p.i. after which viral proteins were immunoprecipitated from the culture media as described before [8] using the anti-TGEV serum (for TGEV) or the combination of G73/A40

Fig. 2. Entry of CCoV, FECV, FIPV and TGEV into PKFA-2 cells. PKFA-2 cells cultured on filter supports were infected with the different viruses from the apical or basolateral side at 7 days post seeding. Cells were fixed at $6 \mathrm{~h}$ p.i. and processed for immunofluorescence analysis as described in the text 


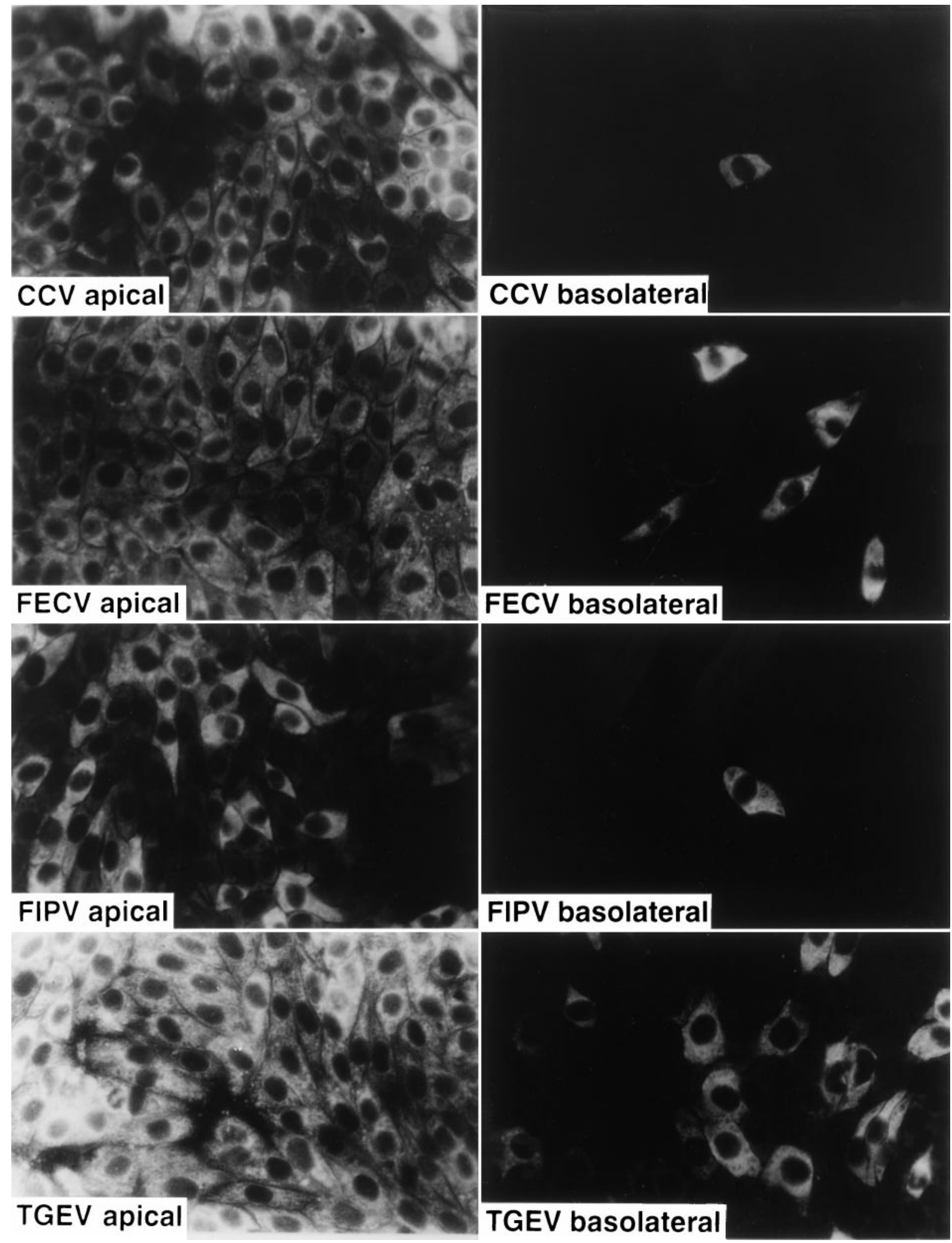




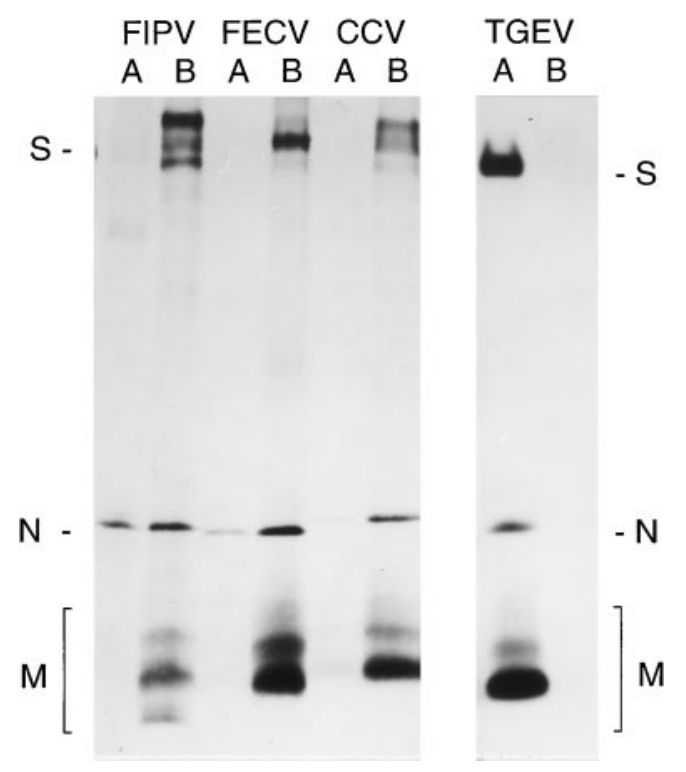

Fig. 3. Release of CCoV, FECV, FIPV and TGEV from PKFA-2 cells. Filter-grown PKFA-2 cells were infected with the different viruses from the apical side at 2 days post seeding. Cells were labelled from 5-8 h p.i. with $200 \mathrm{mCi}^{35} \mathrm{~S}$ amino acids mix. Viral proteins present in the apical $(A)$ and basolateral $(B)$ media were immunoprecipitated as described in the text. The positions of the viral spike $(S)$, membrane $(M)$ and nucleocapsid $(N)$ structural proteins in the gel are indicated on the left and right side of the figure. Note that the protein present at the top of the gel in some of the basolateral samples is an unidentified cellular protein $(\mathrm{Mr} \sim 250 \mathrm{kDa})$ nonspecifically co-immunoprecipitated only from basolateral media of PKFA-2 cells [9]

(for FECV, FIPV and CCoV). Subsequently, the samples were loaded onto an SDS-10\% polyacrylamide gel and electrophoresed. In agreement with the results obtained with the parental LLC-PK1 cells [8], TGEV structural proteins were observed predominantly in the apical medium. To our surprise, however, the structural proteins of FECV, FIPV and CCoV had in each case accumulated on the basolateral side of the cells. The basolateral release of these viruses was confirmed when the titres of virus in the media were determined by performing a limiting-dilution assay ( $n=3$ for each time point; two-fold dilution steps) in PKFA-2 cells. Whereas more than $99 \%$ of the infectious TGEV was released into the apical medium, 87, 90 and 93\% of the CCoV, FECV and FIPV infectious particles, respectively, were found in the basolateral culture medium similar to the release of MHV from LLC-PK1 cells expressing the MHV-receptor [10]. As discussed previously, these latter figures may even be an underestimation of the basolateral release because many particles become trapped between neighbouring cells and between the filter and the cells [9].

In this study, we constructed a cell line (PKFA-2) that stably expressed the recombinant fAPN cDNA by which cells became susceptible to infection by CCoV, FECV and FIPV while maintaining their natural susceptibility to TGEV. FCoVs occur in two serotypes and the FECV and FIPV strains used here are each of type II. It has been reported before that fAPN can also function as a receptor for type I FCoVs [14]. However, we were unable to infect our PKFA-2 cells with the type I FIPV strains UCD-1 and TN406 (results not shown), which is consistent with findings of others [1]. In addition, it was reported recently that a monoclonal antibody against fAPN blocked the infection of Felis catus whole fetus cells by type II but not type I FCoVs [3]. These data imply that type I feline coronaviruses use a receptor that is either different from or required in addition to the fAPN molecule. 
The entry of CCoV, FECV and FIPV occurs preferentially through the apical side of PKFA-2 cells. This is similar to the entry of other coronaviruses, i.e., bovine coronavirus [7, 13], MHV [9] and TGEV [8]. Previously, we have shown that the porcine APN (pAPN) was mainly located on the apical surface of polarized LLC-PK1 cells [8], similar to the apical localization of the human APN in MDCK cells [16]. Conceivably, the fAPN is also located on this side of epithelial cells. As we were mainly interested in virus release, we did not examine this feature further. The observation that the polarity in virus entry appeared to develop only after cells had been grown for longer periods on filters resembles our earlier observations showing that in young monolayers the viral MHV- and TGEV-receptors are still distributed on both sides $[8,10,11]$. With respect to the in vivo situation - coronaviruses are generally transmitted by the fecal-oral and/or the aerogenic routes - apical entry of coronaviruses into epithelial cells seems logical. By entering the organism's respiratory and/or alimentary tracts the viruses first encounter the apical membranes of the epithelial cells that line these cavities. Entry through the apical surface would thus be an efficient way for the viruses to infect these cells, rather than being transported, for instance, by M-cells in the Peyer's patches, to the basolateral surface of the epithelial cells before infection can occur.

As stated earlier, our previous findings made us suggest that the apical release of TGEV and the basolateral release of MHV are essential factors underlying the difference in pathogenesis found between these viruses. However, the apical release of MHV from MDCK cells did not fit our hypothesis. The results of the present study show that this was not an exceptional observation, since the basolateral release of $\mathrm{CCoV}$ and FECV from PKFA-2 cells is also at variance with the hypothesis. Clearly, no direct correlation exists between the sidedness of coronavirus release from epithelial cells and the local or systemic nature of the infection these viruses cause in their host.

Care has to be taken, however, when extrapolating our data to the in vivo situation. As we observed with MHV, the polarity in virus release can be dependent on the type of cell used. Thus, although epithelial cells of the enteric tract and those of the kidney have many features in common, the direction of release of FIPV and FECV from enteric cells (the natural situation) may be different from that in porcine kidney cells.

Whilst the biological relevance of the polar release of coronaviruses remains intriguing, the question of how this specific sorting within the epithelial cells is accomplished still needs to be solved. Coronaviruses are assembled at pre-Golgi membranes to be subsequently exported from the cells following the secretory pathway. In epithelial cells the directional sorting supposedly occurs at the level of the trans-Golgi network. We recently demonstrated that the signals for this sorting are not contained in the viral spike (S) protein: the direction of virus (-like particle) release was independent of the presence of the $S$ protein [12]. It is a challenge for the future to elucidate the sorting signals and mechanisms that effect these processes. 


\section{References}

1. Benbacer L, Kut E, Besnardeau L, Laude H, Delmas B (1997) Interspecies aminopeptidase- $\mathrm{N}$ chimeras reveal species-specific receptor recognition by canine coronavirus, feline infectious peritonitis virus, and transmissible gastroenteritis virus. J Virol 71: 734-737

2. Cerneus DP, Strous GJ, van der Ende A (1993) Bidirectional transcytosis determines the steady state distribution of the transferrin receptor at opposite plasma membrane domains of BeWo cells. J Cell Biol 122: 1223-1230

3. Hohdatsu T, Izumiya Y, Yokoyama Y, Kida K, Koyama H (1998) Differences in virus receptor for type I and type II feline infectious peritonitis virus. Arch Virol 143: 839-850

4. Horzinek MC, Lutz H, Pedersen NC (1982) Antigenic relationships among homologous structural polypeptides of porcine, feline, and canine coronaviruses. Infect Immun 37: $1148-1155$

5. Lai MM, Cavanagh D (1997) The molecular biology of coronaviruses. Adv Virus Res 48: $1-100$

6. Levis R, Cardellichio CB, Scanga CA, Compton SR, Holmes KV (1995) Multiple receptor-dependent steps determine the species specificity of HCV-229E infection. Adv Exp Med Biol 380: 337-343

7. Lin X, O'Reilly K, Storz J (1997) Infection of polarized epithelial cells with enteric and respiratory tract bovine coronaviruses and release of virus progeny. Am J Vet Res 58: 1120-1124

8. Rossen JWA, Bekker CPJ, Voorhout WF, Strous GJAM, van der Ende A, Rottier PJM (1994) Entry and release of transmissible gastroenteritis coronavirus are restricted to apical surfaces of polarized epithelial cells. J Virol 68: 7966-7973

9. Rossen JWA, Voorhout WF, Horzinek MC, van der Ende A, Strous GJAM, Rottier PJM (1995) MHV-A59 enters polarized murine epithelial cells through the apical surface but is released basolaterally. Virology 210: 54-66

10. Rossen JWA, Bekker CP, Strous GJ, Horzinek MC, Dveksler GS, Holmes KV, Rottier PJ (1996) A murine and a porcine coronavirus are released from opposite surfaces of the same epithelial cells. Virology 224: 345-351

11. Rossen JW, Strous GJ, Horzinek MC, Rottier PJ (1997) Mouse hepatitis virus strain A59 is released from opposite sides of different epithelial cell types. J Gen Virol 78: 61-69

12. Rossen JW, de Beer R, Godeke GJ, Raamsman MJ, Horzinek MC, Vennema H, Rottier PJ (1998) The viral spike protein is not involved in the polarized sorting of coronaviruses in epithelial cells. J Virol 72: 497-503

13. Schultze B, Zimmer G, Herrler G (1996) Virus entry into a polarized epithelial cell line (MDCK): similarities and dissimilarities between influenza $\mathrm{C}$ virus and bovine coronavirus. J Gen Virol 77: 2507-2514

14. Tresnan DB, Levis R, Holmes KV (1996) Feline aminopeptidase N serves as a receptor for feline, canine, porcine, and human coronaviruses in serogroup I. J Virol 70: 8669-8674

15. Vennema H, de Groot RJ, Harbour DA, Dalderup M, Gruffydd-Jones T, Horzinek MC, Spaan WJM (1990) Early death after feline infectious peritonitis virus challenge due to recombinant vaccinia virus immunization. J Virol 64: 14071409

16. Wessels HP, Hansen GH, Fuhrer C, Look AT, Sjostrom H, Noren O, Spiess M (1990) Aminopeptidase $\mathrm{N}$ is directly sorted to the apical domain in MDCK cells. J Cell Biol 111: 2923-2930 
17. Yeaman C, Grindstaff KK, Nelson WJ (1999) New perspectives on mechanisms involved in generating epithelial cell polarity. Physiol Rev 79: 73-98

18. Zegers MMP, Hoekstra D (1998) Mechanisms and functional features of polarized membrane traffic in epithelial and hepatic cells. Biochem J 336: 257-269

Author's address: Dr. J. W. A. Rossen, Pediatric Gastroenterology and Nutrition, Rm Ee1512, Erasmus University Medical Center Rotterdam, Dr. Molewaterplein 50, 3015 GE Rotterdam, The Netherlands, e-mail: rossen@kgk.fgg.eur.nl

Received July 24, 2000 